\title{
Utility of serial procalcitonin measurement in management of febrile neutropenia in a resource constrained setting.
}

Rohan Halder ( $\sim$ rohanhalder86@gmail.com)

All India Institute of Medical Sciences https://orcid.org/0000-0002-3100-558X

\section{Tulika Seth}

All India Institute of Medical Sciences

Pradeep Chaturvedi

All India Institute of Medical Sciences

Manoranjan Mahapatra

All India Institute of Medical Sciences

Hara Prasad Pati

All India Institute of Medical Sciences

Seema Tyagi

All India Institute of Medical Sciences

\section{Renu Saxena}

all india institute of medical sciences

\section{Research Article}

Keywords: procalcitonin, febrile neutropenia, invasive fungal infections, serial monitoring

Posted Date: February 19th, 2020

DOI: https://doi.org/10.21203/rs.2.23935/v1

License: (9) This work is licensed under a Creative Commons Attribution 4.0 International License. Read Full License 


\section{Abstract}

Background: Febrile neutropenia is a common cause in morbidity and mortality during treatment of hematological neoplasms.

Methods: Subjects included all cases admitted under hematology department with febrile neutropenia from February to June 2018. Diagnostic work up for each febrile episode was done including Procalcitonin (PCT) was sent at fever onset 0,24,48hour, day 7 and day 14 .

Results: Data was analyzed for 52 febrile episodes in 50 patients.PCT cut off value at 24 hours of $\leq 1.2 \mathrm{ng} / \mathrm{ml}$ had a sensitivity and specificity of $62.5 \%$ and $87.5 \%$ for discriminating IFI and MDI $(p=0.033)$. PCT had a negative predictive value of $70 \%$ for the diagnosis of IFI as compared to MDI. PCT cut-off of $0.4 \mathrm{ng} / \mathrm{ml}$ on day 7 had a sensitivity and specificity of $60.98 \%$ and $100 \%$ respectively for discriminating IFI and PUO ( $p=0.0001$ ). There was a significant fall in the level of PCT by day 14 (median fall in PCT of $10.8)$ in the group of patients who had both IFI+ MDI when compared to other groups $(p=0.047)$. PCT level at 24 and 48 hours can be a predictor of severity of infection.

Conclusion: Procalcitonin at 24 hours could discriminate between bacterial and fungal infection and between fever due to fungal infection and disease fever.

\section{Introduction}

Febrile neutropenia (FN), as defined by the Immunocompromised Host Society (ICHS) [1], is either presence of fever with an absolute neutrophil count (ANC) of $<500$ cells $/ \mathrm{mm} 3$ or presence of fever with an ANC of $<1000$ cells $/ \mathrm{mm} 3$ with an expected decline to $<500$ cells $/ \mathrm{mm} 3$. Febrile neutropenia (FN) is a frequent complication seen in hematological malignancies and this warrants prompt institution of antibiotics. Sometimes it is seen secondary to the hematological disease itself and frequently in the patients on chemotherapy. Often, the etiology of fever is difficult to ascertain in acute leukemia patients. According to European Society of Medical Oncology (ESMO) Clinical Practice Guidelines, positive microbiological culture is found only in $7-31 \%$ of febrile episodes [2]. Also, the clinical criteria for systemic inflammatory response syndrome (SIRS) may fail due to the presence of neutropenia [2]. Without early adequate treatment, the disease may become rapidly fatal [3].

Several studies in ICU settings have highlighted the usefulness of PCT measurement for early and prompt diagnosis of infection [4], characterizing the severity of underlying illness [5, 6], guiding therapy [7-9], and predicting outcome [10-12].

In hemato-oncology setting, larger studies of serial measurements of PCT in febrile neutropenic patients are lacking and most are inconclusive because of small sample sizes and different PCT cut-off values employed.

\section{Materials And Methods}


It was a prospective observational study conducted in the Department of Hematology and Reproductive Biology, AlIMS, New Delhi from February 2018 to July 2018. Patients admitted with hemato-lymphoid malignancies or aplastic anemia with febrile neutropenia (ANC $<500 / \mathrm{mm} 3$ ) aged 1 year to 60 years.

A written informed consent will be obtained from all the included patients/ patients' parents (in case of minor) prior to the study as mandated by the institute ethics committee.

Fever was defined as a single oral temperature measurement of $>38.3 \mathrm{C}(101 \mathrm{~F})$ or a temperature of $>38.0 \mathrm{C}(100.4 \mathrm{~F})$ sustained over a 1-h period. A new febrile episode was defined as a relapsing fever after more than 72 hours of apyrexia $\left(>38^{\circ} \mathrm{C}\right)$. Neutropenia was defined as an ANC of, $<500$ cells $/ \mathrm{mm} 3$ or an ANC that is expected to decrease to, 500 cells/mm3 during the next $48 \mathrm{~h}$.

Febrile episodes were classified according to definitions of the International Immunocompromised Host Society (ICHS):

1. Microbiologically documented infections -based on a positive culture of urine, faeces, throat, bronchoalveolar lavage, CSF or wound- classified as with bacteremia (MDI-B) or without bacteremia (MDI-NB).

Bacteremia will be defined as fever with positive with positive blood cultures for bacteria (peripheral blood or from central venous catheter) with or without septic symptoms

2. Clinically documented infections (CDI), where physical and radiological findings are compatible with an underlying infection

Standard definitions (CDC and WHO) were used for CDI classification.

3. Fever of unexplained origin (FUO) (fever without a clinically documented site of infection and microbiologically documented cause of infection)

4. Invasive fungal infections (IFI) were classified according to the definitions of the EORTC-MSG.

Diagnostic workup for each Febrile Episode included thorough physical examination, blood culture at fever onset drawn by phlebotomy or central venous catheter, urine culture, and chest X-ray.

Additional specific investigations were performed according to clinical presentation oropharyngeal swabs in patients with severe mucositis. In persistent fever for more than 4 days, diagnostic reassessment includes repeat blood culture and serum galactomannan at 72 hours, then day 7 of admission, weekly measurement of galactomannan antigenemia, a thoraco-abdominal CT-scan, a bronchoscopy with BAL if indicated and biopsy for culture and histopathology at any clinically suspected site of infection.

\section{Procalcitonin Measurement}

- During each febrile episode, blood samples for PCT was sent at the same time as blood cultures at the onset of fever before starting I.V. antibiotics and thereafter at $24 \mathrm{hr}, 48 \mathrm{hr}$, day 7, day 14 or till 
resolution of fever whichever is later and at the end of antibiotics ( 6 times for each febrile episode/ patient).

\section{Statistical Analysis}

- Statistical analysis was done using SPSS statistics version 20.0. Chi- square test or fisher exact test was used to compare categorical data. Continuous variables were compared using the MannWhitney (2-group comparison) or Kruskall Wallis (multiple-group comparison) non-parametric tests. The diagnostic property of PCT for different etiologies of fever will be assessed using Receiver operating characteristic analysis (ROC). $P$ value $<0.05$ is considered as statistically significant.

\section{Results}

A total of 72 patients were evaluated out of which 17 patients were excluded because the fever was due to other clearly identified non- infectious causes like blood transfusion, ATRA syndrome/ Drugs. 5 patients were excluded due to incomplete data. Data was analyzed for 52 febrile episodes in 50 patients.

Febrile events were classified into four categories based on clinical and microbiological findings: microbiologically documented infection (MDI); clinically documented infection (CDI); proven or probable invasive fungal disease (IFI); fever of unknown origin (FUO).

Patients demographic characteristics and primary diagnosis at the time of admission were comparable across the four groups. There are a number of patients who had dual infections like CDI and MDI or MDI

and IFI they have been included in both the columns. Most of the patients were evaluated during their $1^{\text {st }}$ febrile episode. (Table 1,2)

Microbiologically documented infection with Bacteremia (MDI-B) was identified in $38.46 \%$, Clinically documented infections in $48 \%$, PUO in $15.38 \%$ and IFI was seen in $28.85 \%$ of all the subjects. The most frequently isolated organism in MDI-B group was Klebsiella pneumonie (8/20 patients) (Table 3).

$35 \%$ of patients in the MDI- B group developed septic shock. There were $9(17.3 \%)$ patients who had both $\mathrm{MDI}$ as well as CDI. And there were 3(5.7\%) patients with mixed infection (i.e. MDI+IFI). Most common CDI was soft tissue infection followed by NEC and perianal infection. When comparing the three groups, IFI, CDI, PUO (disease fever) and MDI-B with respect to duration of neutropenia, duration of hospitalization and time to defervescence there was no significant difference. The duration of antibiotics administration was significantly longer in the CDI group.

\section{Procalcitonin Changes}

PCT kinetics were studied over a period of 14 days during febrile episode of different etiologies. At fever onset (day 0 ) no significant difference was observed in the PCT values among all groups. The median PCT value at 24, 48 hours and 7 days was non-significantly higher in the MDI-B group when compared with other groups. 
There was a significant fall in the level of procalcitonin in the patient who had MDI \& IFI at day 14 (median fall in PCT of 10.8) when compared to the other groups $(p=0.047)$.

The clinically documented infections were further divided into 4 subgroups i.e. line site infections, Neutropenic enterocolitis, Pneumonia, Perianal infection and soft tissue infections (abscesses). On interalia analysis of these subgroups procalcitonin was significantly higher in the pneumonia group when compared to the rest (median-11.42; range-6.170 - 13.003; $p<0.05$ ). A non- significant fall in the procalcitonin value at 48 hours was seen in the perianal infection group when compared to the rest of the subgroups of CDI.

When comparing the PCT values across the phases of chemotherapy most of the cases were in the induction chemotherapy group, PCT values at the onset of fever at (0 hour) and on day 7 were significantly higher in the group undergoing Induction 2(salvage) chemotherapy.

\section{Procalcitonin in septic shock}

The median PCT value at 24 and 48 hours of febrile neutropenia was significantly higher in the group of patients who developed signs of severe infection like Septic shock, hypoxia ( $p$ value- 0.007 for PCT 24 and 0.001 for PCT 48).

The median PCT values were higher on day 7 and day 14 when comparing febrile events due to infectious etiology against disease fever/ Pyrexia of Unknown Origin (PUO). However, the PCT values were not different at fever onset but the values were non-significantly higher at 24 and 48 -hour of the febrile episode.

\section{Procalcitonin Dynamics}

The peak PCT values at various time points were plotted on a line diagram for three groups IFI, CDI and MDI-B and it is seen that the peak values were highest in MDI-B when compared to the other two groups (Figure 1; panel a). The peak PCT trend was plotted for IFI vs other groups \& no significant difference was noted (CDI \& MDI-B) (Figure 1; panel b). Amongst the various CDIs the PCT peak values were highest for the patients who had pneumonia followed by soft tissue infection (Figure 1; panel C). As seen from the figure below (Figure 1; panel d) the PCT values at various time points were non significantly higher in patients with bacteremia when compared to patient with IFI. As seen from the graph below patients with only MDI infections had the highest PCT level over the course of febrile episode when compared to the groups with combined infections or IFI or CDI (Figure 2).

\section{Evaluating the diagnostic performance of PCT}

\section{ROC curve at PCT-24}

After performing ROC, cut off point of PCT at 24 hours (Figure 3a) was $\leq 1.2$ with AUC of .766 and sensitivity and specificity of $62.5 \%$ and $87.5 \%$ respectively for discriminating IFI and MDI which was 
statistically significant with $p$ value $=.0332$. PCT had a $70 \%$ negative predictive value for the diagnosis of invasive fungal infections when compared to other microbiologically documented infection- bacteremia (MDI-B).

That means if PCT at 24 hours was less than or equal to 1.2 then there are significantly higher chances of presence of IFI in the patient as compared to MDI-B.

When comparing the PCT values for invasive fungal infections versus other bacterial infections (MDI/CDI), there was no significant difference in the two groups.

The ROC curves for PCT at other time points were not statistically significant.

After performing ROC, cut off point of PCT at Day 7(Figure 3b) was $>0.4$ with AUC of . 813 and sensitivity and specificity of $60.98 \%$ and $100 \%$ respectively for discriminating IFI and PUO (disease fever) which was statistically significant with $p$ value $=.0001$. It means if PCT at Day 7 of fever was more than or equal to 0.4 then there are significantly higher chances of fever being due to IFI in the patient as compared to disease fever (PUO). ROC curves for IFI vs MDI for PCT at Ohr of fever and 48 hours of fever has been shown below.

\section{Diagnostic performance of PCT for infectious versus non-infectious etiology of fever}

The cut-offs obtained for PCT draws at 0, 24 and 48 hours were not statistically significant. However, the PCT cut-off of $0.4 \mathrm{ng} / \mathrm{ml}$ on day 7 had a sensitivity, specificity, NPV and PPV of $60.98 \%, 100 \%, 27.3 \%$ and $100 \%$ respectively with AUC 0.813 ( $p<0.0001$ ). Also, the PCT values cut-off of 0.32 on day 14 of febrile episode had sensitivity of $48.65 \%$, specificity of $100 \%$, PPV $100 \%$ and NPV $24 \%$ with AUC 0.723 $(p<0.0009)$.

\section{Discussion}

Procalcitonin has been studied as a marker of infection to in patients of febrile neutropenia. To the best of our knowledge, we are aware of only 4 studies who have studied procalcitonin serially (Table 4). Each of those studies had a different design and hence their outcomes too have been different. Also, the cut offs for PCT in each of these studies have been different.

Most of our patients were high risk subjects of febrile neutropenia which is supported by the fact that $77 \%$ of our febrile episodes included patients of acute leukemia. We purposefully excluded patients who had fever during ATG therapy and Hematopoietic stem cell transplant (HSCT) patients as we were aware of reports ATG leading to falsely high PCT values. Hatzistilianou et al study included only children with acute lymphoblastic leukemia. Whereas, Fleishhack et al and Reitmann et al have included not only neutropenic children but also non-neutropenic children in their study to compare the levels between the two groups. Robinson et al have included patients with autologous HSCT subjects as well in their study [9]. The median age of the cohort in our study was 46.5 years which is much less when compared to the 
cohort in Robinson et al where the median age of the subjects was 57 years [9]. One plausible explanation for this observation could be because of $46 \%$ febrile episodes in their study included subjects of myeloma/lymphoma which is usually the disease of the elderly.

We had a significant burden of gram-negative infections with most common being Klebsiella pneumonia $(37.5 \%)$ and significant number of patients fell into 2 or more groups of infections. E. coli $(27.5 \%)$ followed by other gram-negative bacteria (Acinetobacter spp., Pseudomonas) were the most common organism. [17-18] followed by gram positive organisms. This is comparable to the data that was published. But in the study by Rasmy et al, it is quiet contrasting where they found gram positive organisms to be the predominant bacteria responsible for febrile neutropenia [19].

The median duration of neutropenia in our study was 36 days (range=2-400) and the median duration of hospitalization prior to febrile event in our study was 15 (range=2-90) days which contrasts with the study by Robinson et al. whose median duration of neutropenia was 15 days and duration of hospitalization was about 28 days [9]. Probable reason for this observation could be referral bias among patients of Aplastic anemia and acute leukemia.

$27 \%$ of the febrile episodes were the $2^{\text {nd }}$ febrile episode whereas by in the Robinson et al study, $31 \%$ of their febrile events were $2^{\text {nd }}$ febrile episodes. The number of first febrile event and $2^{\text {nd }}$ febrile events are comparable between both the studies. Similar data with respect to febrile episodes are not available in the other two studies.

Hypoxemia requiring mechanical ventilation/ septic shock was seen in $39(69 \%)$ of all the infections. Which contrasts with the study by Robinson et al. where only $2 \%$ of their patients were critically ill. This is most likely because most of our infections were caused due to gram-negative bacteria and they are known to cause severe sepsis.

The median highest PCT values were seen 48 hours after onset of fever in the CDI group. When the CDI was having a $\mathrm{MDI}(\mathrm{n}=9)$ too, the median PCT values were highest on day 14. It means the CDI group responded to the empirical antibiotic regimen and hence within 48 hours the PCT values started to fall. But when CDI was associated with an MDI the antibiotic regimen was not appropriate and hence the PCT values kept rising and had a late response when the antibiotics were changed. Reitman et al compared PCT values which was restricted to studying only bacteremia, they did not include any group with CDI [16].

PCT values in the MDI group peaked on day 7. PCT seems to be an exquisitely specific marker of bacteremia because irrespective of presence of any other cause of fever PCT values were highest on day 7 in MDI when compared to the PUO/CDI (only)/PUO groups. Robinson et al in their study found that the PCT values were significantly higher in non-CNS MDI when compared to all other etiologies of fever [9]. They found that the peak PCT was seen 2 days after onset of fever which was usually the time, they reevaluated their empirical antibacterial therapy. At our center our antibiotic policy is to change the 
antibiotics at 48- 72 hours of fever not responding to empirical antibiotics and the response was seen about 48 to 72 hours after reviewing the first line antimicrobials.

The PCT values in our study rose to their peak at $48 \mathrm{hrs}$ in the IFI group. The peak PCT in the IFI group was not statistically different from the MDI group. Most of the IFI $s$ in our study were of probable fungal infection, proven fungal infections were seen in only 3 cases (all were Aspergillus spp.) and no case of invasive candidiasis was found. A PCT value at 24 hours of $\leq 1.2$ with AUC of 0.766 had a sensitivity and specificity of $62.5 \%$ and $87.5 \%$ for discriminating IFI and MDI $(p=0.033)$. Also, PCT had a negative predictive value of $70 \%$ for the diagnosis of IFI when compared to MDI.

When PCT was compared to PUO at day 7, a value of 0.4 with AUC of 0.813 had a sensitivity and specificity of $60.98 \%$ and $100 \%$ for discriminating IFI and PUO with statistically significant value of 0.0001 .

Robinson et al in their study found that a PCT $>500 \mathrm{pg} / \mathrm{ml}$ beyond 3 days of fever had a sensitivity of $81 \%$, specificity of $57 \%$, PPV $49 \%$, NPV $86 \%$ to diagnose fungal infection and it occurred around day 7 in their study [9].

The MDI+IFI is a unique group which has not been highlighted in other studies. Though the numbers are small $(n=3)$ but this has not been forthcoming in any other studies.

At fever onset the median PCT was highest in this group but it was not statistically significant. There was no statistically significant difference in the PCT values at other time points when compared to other infections.

There was a significant fall in the level of PCT by day 14 in the group of patients who had both IFI+ MDI when compared to other groups $(p=0.047)$ which means that we were able to successfully identify and treat these group of patients. Fleishhack et al. too found in their study that decreasing PCT levels during febrile events reflected defervescence, clinical improvement [14]. Robinson et al. in their study found that a decreasing PCT to normal levels was significantly associated with defervescence and response to antifungal therapy when there was persistently elevated PCT values [9].

The median PCT values were significantly higher in the group undergoing salvage chemotherapy as it is expected that these patients have come with either a bacterial/fungal infection prior to starting chemotherapy and have dysfunctional granulocytes which predispose them to infection. Robinson et al. in their study did not find any significant difference in the PCT values when subjects were stratified to the type of chemotherapy they received [9].

Procalcitonin has been studied as a marker of infection to in patients of febrile neutropenia. To the best of our knowledge, we are aware of only 4 studies who have studied procalcitonin serially (Table 4). Each of those studies had a different design and hence their outcomes too have been different. Also, the cut offs for PCT in each of these studies have been different. 
Most of our patients were high risk subjects of febrile neutropenia which is supported by the fact that $77 \%$ of our febrile episodes included patients of acute leukemia. We purposefully excluded patients who had fever during ATG therapy and Hematopoietic stem cell transplant (HSCT) patients as we were aware of reports ATG leading to falsely high PCT values. Hatzistilianou et al study included only children with acute lymphoblastic leukemia. Whereas, Fleishhack et al and Reitmann et al have included not only neutropenic children but also non-neutropenic children in their study to compare the levels between the two groups. Robinson et al have included patients with autologous HSCT subjects as well in their study [9]. The median age of the cohort in our study was 46.5 years which is much less when compared to the cohort in Robinson et al where the median age of the subjects was 57 years [9]. One plausible explanation for this observation could be because of $46 \%$ febrile episodes in their study included subjects of myeloma/lymphoma which is usually the disease of the elderly.

We had a significant burden of gram-negative infections with most common being Klebsiella pneumonia (37.5\%) and significant number of patients fell into 2 or more groups of infections. E. coli (27.5\%) followed by other gram-negative bacteria (Acinetobacter spp., Pseudomonas) were the most common organism. [17-18] followed by gram positive organisms. This is comparable to the data that was published. But in the study by Rasmy et al, it is quiet contrasting where they found gram positive organisms to be the predominant bacteria responsible for febrile neutropenia [19].

The median duration of neutropenia in our study was 36 days (range=2-400) and the median duration of hospitalization prior to febrile event in our study was 15 (range=2-90) days which contrasts with the study by Robinson et al. whose median duration of neutropenia was 15 days and duration of hospitalization was about 28 days [9]. Probable reason for this observation could be referral bias among patients of Aplastic anemia and acute leukemia.

$27 \%$ of the febrile episodes were the $2^{\text {nd }}$ febrile episode whereas by in the Robinson et al study, $31 \%$ of their febrile events were $2^{\text {nd }}$ febrile episodes. The number of first febrile event and $2^{\text {nd }}$ febrile events are comparable between both the studies. Similar data with respect to febrile episodes are not available in the other two studies.

Hypoxemia requiring mechanical ventilation/ septic shock was seen in 39(69\%) of all the infections. Which contrasts with the study by Robinson et al. where only $2 \%$ of their patients were critically ill. This is most likely because most of our infections were caused due to gram-negative bacteria and they are known to cause severe sepsis.

The median highest PCT values were seen 48 hours after onset of fever in the CDI group. When the CDI was having a $\mathrm{MDI}(\mathrm{n}=9)$ too, the median PCT values were highest on day 14 . It means the CDI group responded to the empirical antibiotic regimen and hence within 48 hours the PCT values started to fall. But when CDI was associated with an MDI the antibiotic regimen was not appropriate and hence the PCT values kept rising and had a late response when the antibiotics were changed. Reitman et al compared 
PCT values which was restricted to studying only bacteremia, they did not include any group with CDI [16].

PCT values in the MDI group peaked on day 7. PCT seems to be an exquisitely specific marker of bacteremia because irrespective of presence of any other cause of fever PCT values were highest on day 7 in MDI when compared to the PUO/CDI (only)/PUO groups. Robinson et al in their study found that the PCT values were significantly higher in non-CNS MDI when compared to all other etiologies of fever [9]. They found that the peak PCT was seen 2 days after onset of fever which was usually the time, they reevaluated their empirical antibacterial therapy. At our center our antibiotic policy is to change the antibiotics at 48- 72 hours of fever not responding to empirical antibiotics and the response was seen about 48 to 72 hours after reviewing the first line antimicrobials.

The PCT values in our study rose to their peak at $48 \mathrm{hrs}$ in the IFI group. The peak PCT in the IFI group was not statistically different from the MDI group. Most of the IFI $s$ in our study were of probable fungal infection, proven fungal infections were seen in only 3 cases (all were Aspergillus spp.) and no case of invasive candidiasis was found. A PCT value at 24 hours of $\leq 1.2$ with AUC of 0.766 had a sensitivity and specificity of $62.5 \%$ and $87.5 \%$ for discriminating IFI and MDI $(p=0.033)$. Also, PCT had a negative predictive value of $70 \%$ for the diagnosis of IFI when compared to MDI.

When PCT was compared to PUO at day 7, a value of 0.4 with AUC of 0.813 had a sensitivity and specificity of $60.98 \%$ and $100 \%$ for discriminating IFI and PUO with statistically significant value of 0.0001 .

Robinson et al in their study found that a PCT $>500 \mathrm{pg} / \mathrm{ml}$ beyond 3 days of fever had a sensitivity of $81 \%$, specificity of $57 \%$, PPV $49 \%$, NPV $86 \%$ to diagnose fungal infection and it occurred around day 7 in their study [9].

The MDI+IFI is a unique group which has not been highlighted in other studies. Though the numbers are small $(n=3)$ but this has not been forthcoming in any other studies.

At fever onset the median PCT was highest in this group but it was not statistically significant. There was no statistically significant difference in the PCT values at other time points when compared to other infections.

There was a significant fall in the level of PCT by day 14 in the group of patients who had both IFI+ MDI when compared to other groups $(p=0.047)$ which means that we were able to successfully identify and treat these group of patients. Fleishhack et al. too found in their study that decreasing PCT levels during febrile events reflected defervescence, clinical improvement [14]. Robinson et al. in their study found that a decreasing PCT to normal levels was significantly associated with defervescence and response to antifungal therapy when there was persistently elevated PCT values [9].

The median PCT values were significantly higher in the group undergoing salvage chemotherapy as it is expected that these patients have come with either a bacterial/fungal infection prior to starting 
chemotherapy and have dysfunctional granulocytes which predispose them to infection. Robinson et al. in their study did not find any significant difference in the PCT values when subjects were stratified to the type of chemotherapy they received [9].

\section{Conclusion}

Procalcitonin can be a useful marker to management of these patients and in guiding antimicrobial therapy. Successful treatment of infection (especially in cases of mixed infection) would lead to rapid fall of PCT levels and persistently high levels indicate need for identifying another source of infection. Procalcitonin at 24 hours of fever could distinguish between bacterial sepsis and invasive fungal infection. Procalcitonin on day 7 of fever was able to distinguish between fever due to malignancy (which is the case on many occasions) and invasive fungal infection. A larger study with daily serial monitoring will be a more definitive study and evaluation of different cut offs for fungal and bacterial infections done in the same patient will be a useful study.

\section{Abbreviations}

ANC- Absolute Neutrophil Count

CDI- Clinically Documented Infections

CRP- $\mathrm{C}$ reactive Protein

ESMO-European Society of Medical Oncology

FN- Febrile Neutropenia

$\mathrm{Hr} / \mathrm{Hrs}-$ Hour/Hours

ICHS- Immunocompromised Host Society

IDSA- Infectious Disease Society of America

IFI/IFD- Invasive Fungal Infections/Invasive Fungal Disease

iNOS- Nitric Oxide Synthase

LPS- Lipopolysaccharide

MDI- Microbiologically Documented Infections

PCT-Procalcitonin

PUO/FUO- Pyrexia of Unknown Origin/Fever of Unknown Origin 


\section{Key Points}

1. PCT values at 24 and 48 hours were significantly higher in patients with septic shock, hypoxia and requiring mechanical ventilation ( $p$ value- 0.007 for PCT 24 and 0.001 for PCT 48).

2. Successful treatment of infection (especially in cases of mixed infection) would lead to rapid fall of PCT levels and persistently high levels indicate need for identifying another source of infection.

3. Procalcitonin at 24 hours could discriminate between bacterial and fungal infection and between fever due to fungal infection and disease fever.

\section{Declarations}

Ethics: The study was approved by the Institute Ethics Committee (IEC) at All India Institute of Medical Sciences, New Delhi date 29.03.2017 with Ref.No. IECPG-6/16.02.2017, RT-16/22.03.2017 under leadership of Dr. Sanjeev Sinha Member-secretary, IEC.

Consent for publication: All the authors consent to publish the article in Indexed journal.

Availability of data and materials: The data published in this study is original to the author and the raw data is available with the first author as he is the primary author of this study.

Disclosures/Conflicts of interest: The authors have no conflicts of interest to disclose.

Funding: Research grant received from Indian Council of Medical Research (ICMR) under Ministry of Human Resource Development letter dated 30.10.2017 bearing No. 3/2/Jan-2017/PG-Thesis-HRD (3). The funding was used to purchase kits for performing the Procalcitonin kits.

Author contribution: All the authors have equally contributed to the manuscript.

Acknowledgement: None

\section{References}

1. Smith TJ, Khatcheressian J, Lyman GH et al (2006) 2006 update of recommendations for the use of white blood cell growth factors: an evidence-based clinical practice guideline. J Clin Oncol 24(19):3187-3205

2. de Naurois J, Novitzky-Basso I, Gill MJ, Marti FM, Cullen MH, Roila F (2010) Management of febrile neutropenia: ESMO Clinical Practice Guidelines. Ann Oncol 2010 (Suppl 5):v252-v256

3. Malik I, Hussain M, Yousuf H (2001) Clinical characteristics and therapeutic outcome of patients with febrile neutropenia who present in shock: need for better strategies. J Infect 2001; 42(2):120-125

4. Simon L, Gauvin F, Amre DK, Saint-Louis P, Lacroix J: Serum procalcitonin and C-reactive protein levels as markers of bacterial infection: a systematic review and meta-analysis. Clin Infect Dis 2004; 39: 206-217. 
5. Barnes C, Ignjatovic V, Newall F, Carlin J, Ng F, Hamilton S, Ashley D, Waters K, Monagle P: Change in serum procalcitonin (deltaPCT) predicts the clinical outcome of children admitted with febrile neutropenia. Br J Haematol 2002; 118: 1197-1198.

6. Brunkhorst FM, Wegscheider K, Forycki ZF, Brunkhorst R: Procalcitonin for early diagnosis and differentiation of SIRS, sepsis, severe sepsis, and septic shock. Intensive Care Med 2000; 26: S148S152.

7. Christ-Crain M, Jaccard-Stolz D, Bingisser R, Gencay MM, Huber PR, Tamm M, Muller B: Effect of procalcitonin-guided treatment on antibiotic use and outcome in lower respiratory tract infections: cluster-randomised, single-blinded intervention trial. Lancet 2004; 363: 600-607.

8. Sandek A, Springer J, Habedank D, Brunkhorst F, Anker SD: Procalcitonin-guided antibiotic treatment in heart failure. Lancet 2004; 363: 1555-1556.

9. Robinson JO, Calandra T, Marchetti O: Utility of procalcitonin for the diagnosis and the follow-up of infections in febrile neutropenic patients. Rev Med Suisse 2005; 1: 878-6.

10. Oberhoffer M, Vogelsang H, Russwurm S, Hartung T, Reinhart K: Outcome prediction by traditional and new markers of inflammation in patients with sepsis. Clin Chem Lab Med 1999; 37:363-368.

11. Luyt CE, Guerin V, Combes A, Trouillet JL, Ayed SB, Bernard M, Gibert C, Chastre J: Procalcitonin kinetics as a prognostic marker of ventilator-associated pneumonia. Am J Respir Crit Care Med 2005; 171: 48-53.

12. Oczenski W, Fitzgerald RD, Schwarz S: Procalcitonin: a new parameter for the diagnosis of bacterial infection in the perioperative period. Eur J Anaesthesiol 1998; 15: 202-209.

13. Verlinden A, Veronique de vroey, Ella Roelant et al. Comparison of the power of procalcitonin and CReactive Protein to differentiate between different etiologies of febrile neutropenia in patients with prolonged profound neutropenia. Blood 2017. 130:3221

14. G.Fleischhack, D.Cipic, J. Juettner, C.Hasan, U.Bode. Procalcitonin \pm a sensitive inflammation marker of febrile episodes in neutropenic children with cancer. Intensive Care Med (2000) 26: S202-S211.

15. Hatzistiilianou M, Rekliti A, Athanassiadou F, Catriu D. Procalcitonin as an early maker of bacterial infection in neutropenic febrile children with acute lymphoblastic leukemia. Inflammation Research 2010:59:339-347

16. Reitman A.J, Pisk RM, Gates III JV, Ozeran JD. Serial Procalcitonin levels to Detect bacteremia in Febrile Neutropenia. Clinical Pediatrics. 51(12) 1175-1183

17. Johnson MP, Ramphal R. Beta-lactam-resistant Enterobacter bacteremia in febrile neutropenic patients receiving monotherapy, J Infect Dis, 1990, vol. 162 (pg. 981-3)

18. Johnson PR, Liu Yin JA, Tooth JA. A randomized trial of high-dose ciprofloxacin versus azlocillin and netilmicin in the empirical therapy of febrile neutropenic patients, J Antimicrob Chemother, 1992, vol. 30 (pg. 203-14)

19. Rasmy A, Amal A, Fotih S, Selwi W. Febrile Neutropenia in Cancer Patient: Epidemiology, Microbiology, Pathophysiology and Management. J Cancer Prev Curr (2016) Res 5(3): 00165. 


\section{Tables}

Table 1: Table showing various groups and their age distribution.

\begin{tabular}{|c|c|c|c|c|c|c|}
\hline \multirow[b]{2}{*}{ Age distribution } & \multicolumn{4}{|l|}{ Group } & \multirow[b]{2}{*}{ Total } & \multirow[b]{2}{*}{ P value } \\
\hline & Clinical & IFI & MDI & PUO & & \\
\hline 1-10yrs & $4(16.00 \%)$ & $0(0.00 \%)$ & $4(20.00 \%)$ & 1 (12.50\%) & 9 (14.06\%) & 0.890 \\
\hline 11-20yrs & $6(24.00 \%)$ & 3 (27.27\%) & $3(15.00 \%)$ & $3(37.50 \%)$ & 15 (23.44\%) & \\
\hline 21-30yrs & $8(32.00 \%)$ & $4(36.36 \%)$ & $5(25.00 \%)$ & $2(25.00 \%)$ & 19 (29.69\%) & \\
\hline 31-40yrs & $2(8.00 \%)$ & $0(0.00 \%)$ & $1(5.00 \%)$ & $1(12.50 \%)$ & $4(6.25 \%)$ & \\
\hline 41-50yrs & $4(16.00 \%)$ & 2 (18.18\%) & $4(20.00 \%)$ & $0(0.00 \%)$ & 10 (15.63\%) & \\
\hline$>50 y r s$ & $1(4.00 \%)$ & $2(18.18 \%)$ & $3(15.00 \%)$ & $1(12.50 \%)$ & 7 (10.94\%) & \\
\hline Total & 25 (100.00\%) & $11(100.00 \%)$ & 20 (100.00\%) & $8(100.00 \%)$ & 64 (100.00\%) & \\
\hline
\end{tabular}

Table 2: Groups according to the diagnosis.

\begin{tabular}{|c|l|l|l|l|l|l|}
\hline \multirow{2}{*}{ Primary diagnosis } & \multicolumn{2}{|l|}{ Group } & \multirow{2}{*}{ PUO } & Total \\
\cline { 2 - 5 } & Clinical & IFI & MDI & P value \\
\hline Aplastic Anemia & $1(4.00 \%)$ & $1(9.09 \%)$ & $3(15.00 \%)$ & $0(0.00 \%)$ & $5(7.81 \%)$ & 0.314 \\
Acute Lymphoblastic Leukemia & $5(20.00 \%)$ & $6(54.55 \%)$ & $4(20.00 \%)$ & $4(50.00 \%)$ & $19(29.69 \%)$ \\
Acute Myeloid Leukemia & $17(68.00 \%)$ & $4(36.36 \%)$ & $11(55.00 \%)$ & $4(50.00 \%)$ & $36(56.25 \%)$ \\
Non -Hodgkin Lymphoma & $2(8.00 \%)$ & $0(0.00 \%)$ & $2(10.00 \%)$ & $0(0.00 \%)$ & $4(6.25 \%)$ \\
Total & $25(100.00 \%)$ & $11(100.00 \%)$ & $20(100.00 \%)$ & $8(100.00 \%)$ & $64(100.00 \%)$ \\
\end{tabular}

Table 3: Various microorganisms isolated in the present study. 


\begin{tabular}{|c|c|c|c|c|c|}
\hline \multirow{2}{*}{$\begin{array}{l}\text { Microbiologically Documented Infections with } \\
\text { Bacteremia }\end{array}$} & \multicolumn{3}{|l|}{ Group } & \multirow[b]{2}{*}{ Total } & \multirow{2}{*}{$\begin{array}{l}P \\
\text { value }\end{array}$} \\
\hline & Clinical & IFI & MDI & & \\
\hline Acinetobacter baumanii & $\begin{array}{l}1 \\
(11.11 \%)\end{array}$ & $0(0.00 \%)$ & $1(5.00 \%)$ & $2(6.25 \%)$ & 0.944 \\
\hline Coagulase Negative Staphylococcus & $\begin{array}{l}2 \\
(22.22 \%)\end{array}$ & $0(0.00 \%)$ & $2(10.00 \%)$ & $4(12.50 \%)$ & \\
\hline Enterococcus faecium & $\begin{array}{l}1 \\
(11.11 \%)\end{array}$ & $0(0.00 \%)$ & $1(5.00 \%)$ & $2(6.25 \%)$ & \\
\hline Escherechia Coli & $\begin{array}{l}2 \\
(22.22 \%)\end{array}$ & $\begin{array}{l}1 \\
(33.33 \%)\end{array}$ & $6(30.00 \%)$ & $9(28.12 \%)$ & \\
\hline Klebsiella Pneumonia & $\begin{array}{l}2 \\
(22.22 \%)\end{array}$ & $\begin{array}{l}2 \\
(66.67 \%)\end{array}$ & $8(40.00 \%)$ & $12(37.50 \%)$ & \\
\hline Methicillin Resistant Staphylococcus Aureus & $0(0.00 \%)$ & $0(0.00 \%)$ & $1(5.00 \%)$ & $1(3.13 \%)$ & \\
\hline Pseudomonas & 1 & $0(0.00 \%)$ & $1(5.00 \%)$ & $2(6.25 \%)$ & \\
\hline Aeruginosa & $(11.11 \%)$ & & & & \\
\hline Total & $\begin{array}{l}9 \\
(100.00 \%)\end{array}$ & $\begin{array}{l}3 \\
(100.00 \%)\end{array}$ & $\begin{array}{l}20 \\
(100.00 \%)\end{array}$ & $\begin{array}{l}32 \\
(100.00 \%)\end{array}$ & \\
\hline
\end{tabular}

Table 4: comparison with similar other studies on procalcitonin.

\begin{tabular}{|l|l|l|l|l|}
\hline & Sample size (n) & Age group (years) & Procalcitonin frequency & $\begin{array}{l}\text { Comparator biomarker } \\
\text { (if any) }\end{array}$ \\
\hline Reitman et al. [44] & 89 & $0.3-18$ & 2 readings 12-24 hrs apart & nil \\
\hline Hatzistilianou et al. [43] & 67 & $<18$ & 7 readings / 7 days & CRP, IL-1b, IL-8, TNF-R, \\
\hline Fleischhack et al. [26] & 208 & $<18$ & Daily till resolution of fever & CRP \\
\hline Robinson JO et al. [9] & 194 & $>18$ & $0,1,2,3,4$ and 7 & nil \\
\hline Verlinden et al. [23] & 93 & $>18$ & Daily till fever resolution & CRP \\
\hline Current study & 52 & $3-80$ & $0,1,2,7,14$ (or resolution of fever) & nil \\
\hline
\end{tabular}

\section{Figures}



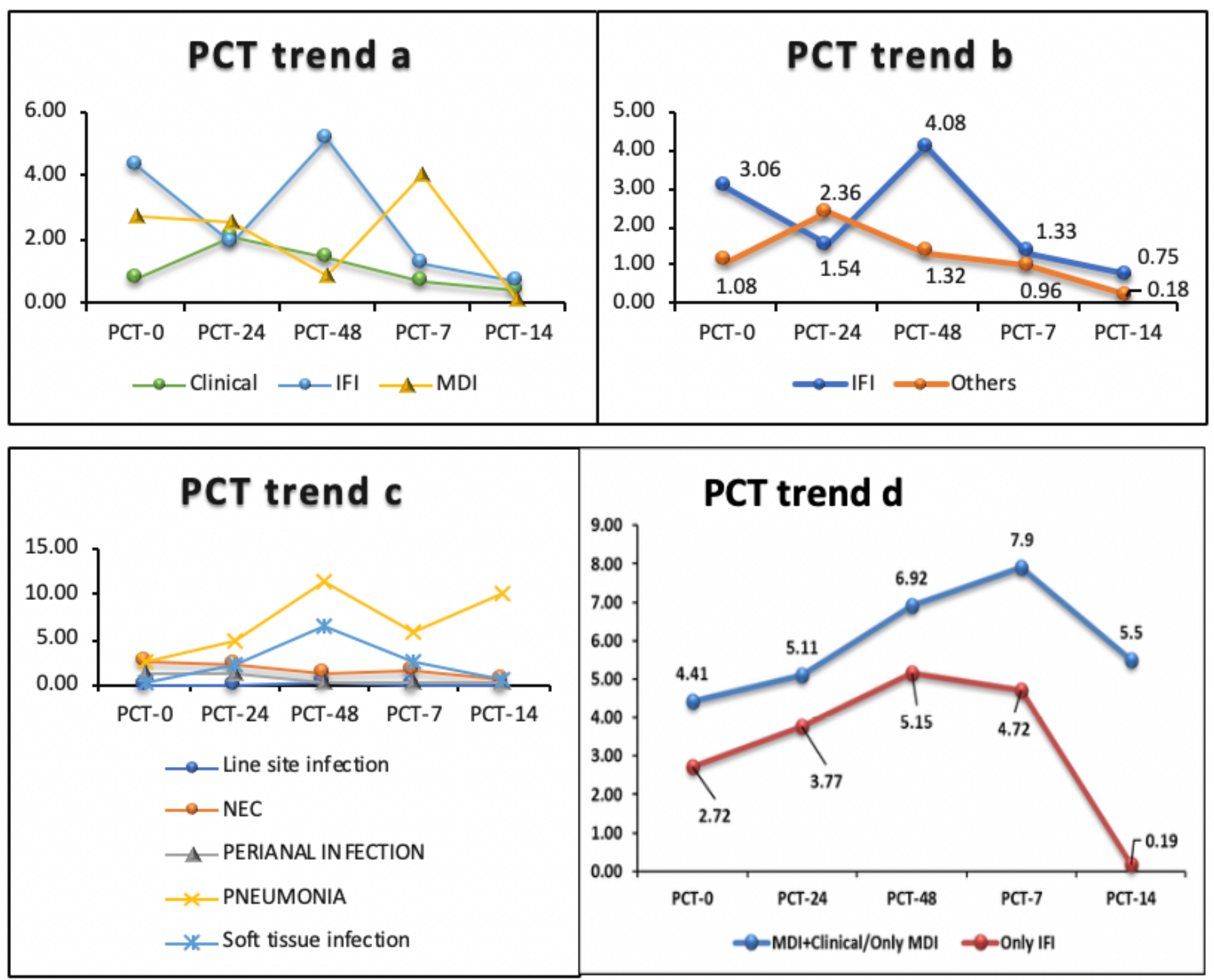

Figure 2

a: PCT trends at the time point measured amongst Invasive fungal infections, Clinically documented infections and Microbiologically documented infections. b: Comparing PCT trends between IFI and Other groups (CDI/MDI etc). C: PCT dynamics between the various group of Clinically documented infections d: PCT dynamics in bacteremia and IFI. 


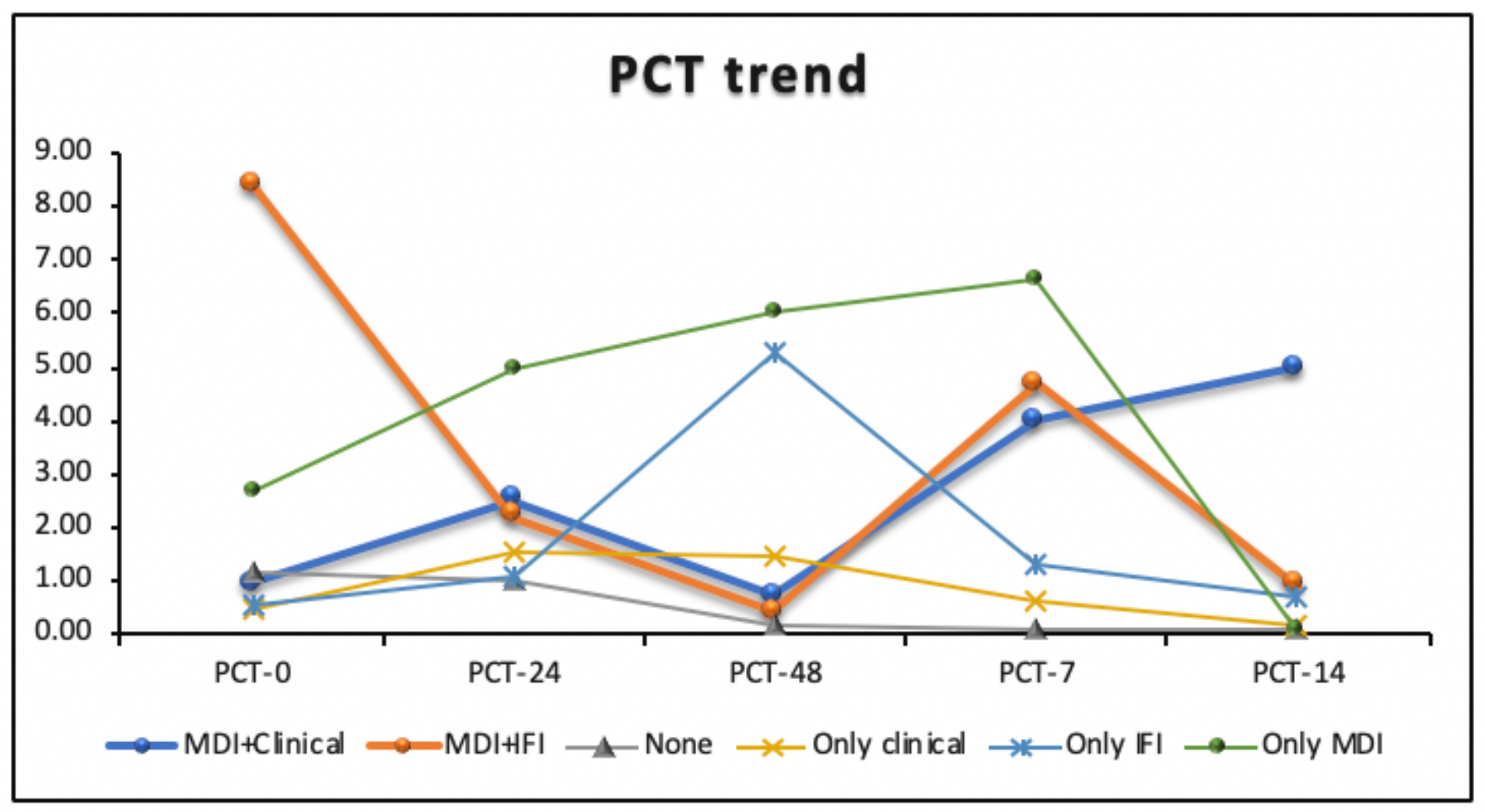

Figure 4

PCT dynamics at various time points between different infections.
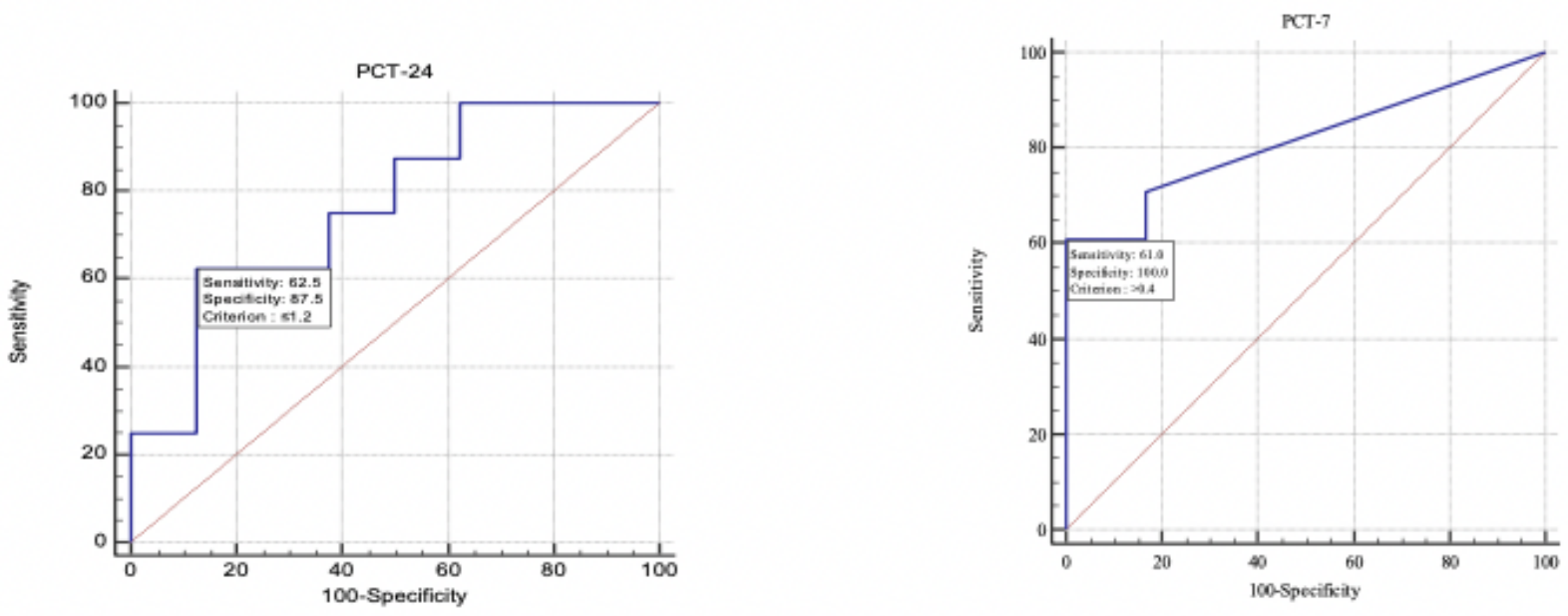

Figure 5

a: and b: Receiver operating curve for PCT at 24 hours and on Day 7. 